\title{
A multiobjective analysis of the potential of scheduling electrical vehicle charging for flattening the duck curve
}

\author{
Raka Jovanovic* \\ Qatar Environment and Energy Research Institute, Hamad bin Khalifa University, PO Box 5825, Doha, \\ Qatar \\ Sertac Bayhan \\ Qatar Environment and Energy Research Institute, Hamad bin Khalifa University, PO Box 5825, \\ Doha, Qatar \\ I. Safak Bayram \\ Department of Electronic and Electrical Engineering, University of Strathclyde, 204 George St., Glasgow, \\ G1XW, United Kingdom
}

\begin{abstract}
In this paper, we investigate the demand-flexibility of large-collections of electric vehicles (EVs) by scheduling their demand to flatten the electricity duck curve that emerge as a result of growing solar power production. The scheduling problem is investigated in a bi-objective setting and an additional objective function related to the amount of charge provided to EVs is also analyzed. The first objective is the minimization of the ramp-up requirements of the system. The second objective reflects the quality of service and the potential level of charging station's profit margins. An important characteristics of the proposed model is the effect of total charging capacity on the two objective functions. The analysis is carried out based on a quadratic programming model which is used to calculate the Pareto Front of the two objective functions. This is done through a case study based on real-world data for EV driver behavior, solar generation, and energy consumption. The computational experiments show that there is a high level of competition between these two objectives. Moreover, the effect of different maximal charging capacities on these objectives is observed.
\end{abstract}

\footnotetext{
* Raka Jovanovic

Email address: rjovanovic@hbku.edu. qa (Raka Jovanovic)
} 
Keywords: Duck curve, Scheduling of charging demand, Electric vehicles, Multiobjective, Quadratic programming

\section{Introduction}

In the past several years, there has been an immense increase in the use of solar energy for electricity production. Even though renewable generation reduces carbon emissions and improves air quality, the power grid operators face operational challenges with high solar penetration. The main one is related to the imbalance between peak electricity demand and renewable energy production during afternoons, resulting in the "duck curve" issue. As the difference between peak demand and production from non-renewables increase, power system operators need to ramp up their production capacity, which leads to financial losses [1, 2]. As the share of renewable solar increases, it is expected that "duck curves" will be more problematic.

In parallel, many governments have provided significant incentives for electric vehicle (EV) adoption to meet net-zero emission goals. This has resulted in a staggering growth in the rate of EVs adoption, and it is expected that in the next few decades, EVs will become a primary mode of ground transportation. These new developments have resulted in the emergence of a new field in research that is dedicated to evaluating the potential of exploiting EV demand flexibility to address congestion issues and release additional capacity, which in turn enables higher connection of low carbon technologies such as EVs and renewables. For the case of EVs, this is often done through scheduling the charging of large groups of vehicles, in a way that minimizes the rampup requirements of non-renewable power generation. In addition to EVs, residential and industrial demand response programs, as well as energy storage technologies are used to achieve this goal[3].

EV charging infrastructures can be divided into three groups. The first one is fast charging which can fill up a typical EV battery in less than 30 minutes with a $50+\mathrm{kW}$ charger. Second option is to charge vehicles at home typically during night time with 2-3 kW residential chargers. However, none of the charging options are suitable for smoothing duck curves since the charging duration is very short for fast charging and 
there is no solar generation during night time charging. Therefore, optimal charging of EVs located at parking lots (e.g. workplace, university, etc.) with level 2 chargers (5-7 kW) can effectively contribute to reduction in power system ramping requirements [4, 5]. Over the last years, there is a growing body of literature on optimal scheduling of EVs located in parking lots [6, 7, 8]. For example, small-scale PV charging systems can effectively lower charging demand and increase carbon savings [9]. Research has been dedicated to exploring a wide range of other benefits of using smart scheduling of EV charging at the workplace [10, 11, 12] and commercial [13] parking lots. The potential of scheduling EV charging to lessen the duck curve has also been assessed in the context of park-and-ride facilities [14].

Optimal demand-side scheduling approach has also been applied to home appliances and thermostatically controlled loads for lowering peak demand [15, 16]. However, the demand flexibility potential of EVs is much higher due to the size, aggregate load, and the duration of EV charging. Some examples of EV demand scheduling can be found in [17], [18], and [19]. Some important new issues that arise in EV charging systems are connected to the amount of satisfied charging requests [20, 21]. When designing such systems, the choice of an adequate maximal charging capacity (MCC) of the facility is of utmost importance due to its relation to the operations of electric distribution systems and infrastructure costs [22, 23, 24].

In the majority of published research, the analysis of positive effects of scheduling EV charging are observed from a single objective perspective, usually minimizing ramp-up requirements or peak loads. In this paper, the focus is on a more holistic approach. To be exact, we explore the relationship between the minimization of ramp-up requirements and the level of satisfied EV charge requests. In addition, this relation is examined for different values of the MCC. To achieve this, a quadratic programming model is developed.

Another issue with existing literature is the lack of use of real-world data for charg55 ing sessions of individual EVs. This is, to a large extent, a consequence of the absence in availability of such data sets. In [25] and [26], data collected for a Dutch smart EV charging provider ElaadNL was used. In [27], data collected from a Chinese charging system was adopted, while in [28] data from charging network in Los Angeles was 
used. The problem with these data sets is difficult access for the wider research community. This gap has recently been filled by researchers from the California Institute of Technology (Caltech) by providing the ACN-Data [21] set that provides the information on a large number of individual EV charging sessions. It should be noted that similar data can be found for residential EV charging through the Pecan Street Dataport [29] that is publicly available for academic research. Since this paper considers public charging, the ACN-Data is used to generate case studies.

The paper is organized as follows. The next section introduces the quadratic programming model used for finding the optimal schedules for EV charging. The third section gives details on the method used for generating test instances for the case study. In the fourth section, the results of the conducted computational experiments are given. This section is divided into three subsections. The first one focuses on the effect of different MCCs; the second one, on the relation between these values and the duck curve, and the third one explores the link between ramp-up requirements and the amount of satisfied charging requests.

\section{Mathematical Model}

In this section, the proposed quadratic program $(\mathrm{QP})$ is presented in detail. The model extends the one presented in [21]. The main differences are the consideration of maximal charge of the station at each time period, minimal amount of charge received by an EV and a new objective focused on maximizing the total charge provided to EVs visiting the facility. The proposed model is based on the following assumptions. The scheduling is done over a predefined time window, which is divided into time periods of equal length. It is assumed that EVs arrive at the charging station (office parking) in different time periods. The arrival and departure time of each EV are known in advance. An EV can be charged during all time periods at the charging station and the charge rate can be adaptable. Upon arrival, each EV requests a specific amount of charge that it wishes to receive. It is further assumed that the maximal charging rate of the EV is higher that the power of the chargers used in the station. Consequently, the charging rate is independent of the vehicle being charged. The base load, the total 
electricity consumption minus renewable generation, are known for each time period.

The proposed QP model is designed in a bi-directional setting. The goal of the model is to find a schedule for EV charging with the following requirements. The first one is related to the power system operator who wishes to avoid periods with high ramping requirements. The second goal, on the other hand, is related to EV drivers who wish to maximize the amount of energy transferred to EVs. The parameters used in the model are given as below:

- The total number of time periods is $T$, and a corresponding set $\mathcal{T}=\{1 \ldots T\}$ denotes all time periods.

- The total number of EVs that visit the charging station is $N$. In addition, a set $\mathcal{E}=\{1 . . N\}$ of all EVs is defined.

- Parameters $a_{i} / d_{i}$ are defined for each $i \in \mathcal{E}$, and provide the arrival / departure time period for an EV $i$.

- A parameter $m_{t}$ is defined for each $t \in \mathcal{T}$, which provides the maximal charging capacity of the charging station. To be more precise, $m_{t}$ is the upper bound for the amount of charge that the station can provide at time period $t$ to the EVs. For the sake of simplicity, a unified MCC $m$ has been used for all time periods.

- For each time period $t \in \mathcal{T}$ a parameter $b_{t}$ specifies the base load of the system. It is equal to the total electric demand minus renewable generation of the system at the time period $t$.

- For an $\mathrm{EV} i \in \mathcal{E}$, a the parameter $k_{i}$ is equal to the requested amount of charge.

- The real parameter $\alpha$ is used for the minimal relative charge an EV must receive compared to the requested one. The same $\alpha$ value, between 0 and 1, is used for all EVs.

- The real parameter $r$ is used for the maximal amount of charging that the facility can provide to a single EV at each time period. This constraint is directly connected to the level 2 charger power. 
In addition to the parameters given above, the following set of decision variables are defined for specifying the optimal scheduling:

- For each EV $i \in \mathcal{E}$ and time period $t \in \mathcal{T}$ a real variable $r_{i t}$ is defined and represents the level of charge received by EV $i$ at time period $t$. Note the modern EV chargers are capable to deliver power at a certain level instead of legacy EV charger's turn on/off capability, consequently a binary variable would not be sufficient.

- For each time period $t \in \mathcal{T}$ an auxiliary real variable $l_{t}$ is defined to denote the total charging received by EVs at time period $t$.

- For each time period $t \in \mathcal{T}_{0}$, where $\mathcal{T}_{0}=\mathcal{T} \backslash\{1\}$, an auxiliary real variable $c_{t}$ is defined to represent the difference in load (the sum of charging load and base load) between two consecutive time periods.

Recall that the focus of this work is on the relation between the goals of the power system operator on the one side and the users of EVs and the charging facilities on the other side. In case of the former, the main objective is the minimization of ramp-up requirements which can be formalized using the following equation:

$$
\text { Minimize } \sum_{t \in \mathcal{T}_{0}} c_{t}^{2}
$$

Eq. 1 simply states that the objective is to minimize the sum of demand changes between consecutive time periods. Instead of using the absolute value of the linear terms $c_{t}$, the quadratic $c_{t}^{2}$ is used with the intention of avoiding steep slopes in the demand curve. Note that the objective of minimizing ramping up requirements is often modeled as the minimization of the maximal change of total demand between two consecutive time periods [14]. The issue with this approach is that it often results in solutions that frequently the sign of the derivative of the function of total demand overtime.

The second goal is related to the preferences of the EV drivers and the charging facility. In this case, the objective is to maximize the total charge received by the EVs, which can be formalized as follows:

$$
\text { Maximize } \sum_{t \in \mathcal{T}} l_{t}
$$


Eq. 2 simply maximizes the sum of received charge $l_{t}$ overall the time periods. The goal of the charging facility is understandably to maximize profits, which is directly related to the total charge provided. At the current state of adoption of EVs, the maximization of profits is equivalent to the level of charge. The main reason for this is a high difference between the cost of a charging $\mathrm{kWh}$ and the price of kWh given by the electric utility company. For example, at California Institute of Technology (Caltech) campus, the unit price electricity $(\mathrm{kWh})$ is around 10 cents while the dynamic price of a $\mathrm{kWh}$ from the distribution company would vary between 1.5 and 2.1 cents. The presented scheduling model is further subject to the following constraints:

$$
\begin{array}{rl}
\sum_{t=a_{i} . d_{i}} r_{i t} \leq k_{i} & i \in \mathcal{E} \\
\sum_{t=a_{i} . d_{i}} r_{i t} \geq \alpha k_{i} & i \in \mathcal{E} \\
r_{i t}=0 & i \in \mathcal{E}, t<a_{i} \\
r_{i t}=0 & i \in \mathcal{E}, t>d_{i} \\
l_{t}=\sum_{i \in \mathcal{E}} r_{i t} & t \in \mathcal{T} \\
l_{t} \leq m & t \in \mathcal{T} \\
c_{t}=l_{t}+b_{t}-l_{t-1}-b_{t-1} & t \in \mathcal{T} 0 \\
0 \leq r_{i t} \leq r &
\end{array}
$$

The constraint given in Eq. (3) states that for each EV $i$, the sum of received charge is less or equal than the requested charge $k_{i}$. Eq. (4) provides that each vehicle will receive a minimal amount of charge which is a certain part of the requested charge. In this equation, $\alpha$ is an input parameter that is used for all the EVs. Eqs. (5) and (6) only allow charging to be done at time periods when an EV $i$ is at the station. Eq. (7) is used for setting the auxiliary variable for the total received charge by EVs at the station at time period $t$. The constraints given in Eq. (8) are used to set a limit on the MCC, maximal amount of charge that EVs can receive at the facility at a time period. Eq. 10 enforces the maximal charge rate of an EV and forbids negative charge. 


\section{Case Study Setup}

Although there has been a significant amount of published research on the scheduling of EV charging to lessen duck curves, the use of real world data has been limited. study setting. One of the main reasons for this is the lack of publicly available data sets. Recently, a research group from Caltech has provided an extensive data set (ACN-Data) containing information about individual charging sessions of EVs for several locations. A detailed description of this data set and examples of its usage can be found at [21]. 
In the proposed model, this is reflected by the number of real world EVs that are presented by a single EV in the model. For example, in an instance with $200 \mathrm{EVs}$, each EV in the model represents $15000 \mathrm{EVs}$ in the real world. The maximal rate of charging for an EV has been assumed to be limited to the charging capabilities of the charger and is set to $7 \mathrm{~kW}$. This value is scaled in the same way as the number of cars. In practice this means that the requested charge $\left(k_{i}\right)$, maximal charging rate for an EV $(r)$ and the maximal charging rate for the facility $(m)$ have been scaled appropriately.

The upper bound for the MCC of the facility is one of the values that is analyzed in the case study, so details for this value are given in the next section. The evaluated time window was a day ( 24 hours). Each time period in the model corresponded to 5 minutes and the value of parameter $T$ is 288 . It should be noted that in late night periods nearly no EVs are at the charging facility. The base load, MCC and maximal charging rate for an EV need to be scaled relative to the length of the time period, which is done in the same way as in [21].

The proposed QP has been implemented using OPL in IBM ILOG CPLEX Optimization Studio Version: 12.6.1.0, and executed using the default solver settings. The Pareto Front has been calculated using the standard $\epsilon$-constraint method [31]. It should be noted that solving a single problem instance to optimality, generally took less than five seconds, in case of calculating the Pareto front multiple such instances needed to be solved.

\section{Case Study}

In this section, a number of computational experiments are presented to analyse the proposed scheduling framework and evaluate its aspects. The first part is dedicated to the influence of the station's MCC on the flattening of a duck curve. In the works of [22, 23, 24], it has been shown that the maximal charging capacity of an EV charging stations is generally limited by the electrical distribution system. The second part of the tests focuses on the effect of different customer satisfaction levels on the shape of the duck curve in relation to the MCC. The final group of tests evaluate the relation of 




(a) Winter

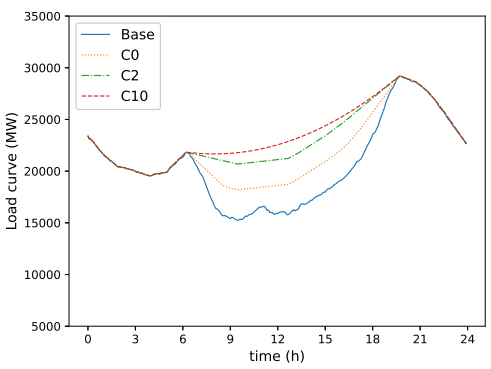

(c) Summer

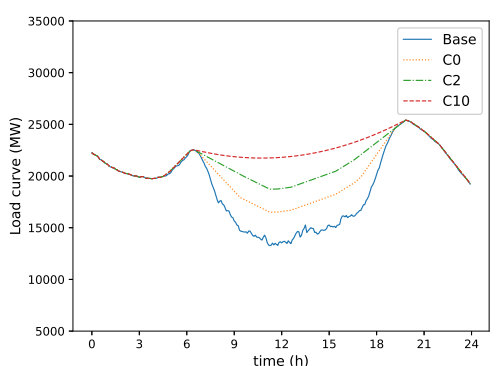

(b) Spring

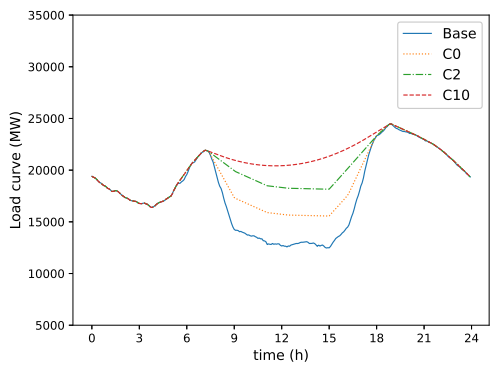

(d) Autumn

Figure 1: Illustration of the maximal levels of flattening of the duck curve that can be achieved for different values of the MCC. Separate subfigures are used for each season.

Pareto Front for the presented quadratic model.

\subsection{Maximal Charging Capacity of the Station}

As previously stated, the MCC of a station is often constrained by the electrical distribution company due to issues related to stability of the system. On the other

Because of this, we analyze the effect of having different levels of MCC in relation to the expected requested charge by the users of the charging facility. The upper bound $U$ for the MCC's is equal to the number of chargers it contains and their capacity. A natural lower bound for the MCC is related to the sum of expected daily charge requests 220

by the users of the facility. To be more precise, the lower bound $L$ is equal to this value divided by the number of time periods over which the scheduling is conducted. 
The difference in these two bounds ( $L$ and $U$ ) can be significant; the upper bound is nearly five times higher than the lower bound for the Caltech data. These values are of course dependent on the occupancy rate of the facility but in case of the ACN data these are the observed values. In the evaluation, the following notation is used:

$$
C_{i}=L+\frac{i}{N}(U-L)
$$

In Eq. 11], $N$ is used to indicate the number of different charging rates that have been evaluated. The notation $C_{i}$ is used for the $i$ th tested MCC, where $i$ is a value between 0 and $N$. In our tests $N$ is set to 10 . Note that, since the $\frac{U}{L}$ is around $5, C_{5}$ will be around three times the minimal charging rate. An evaluation of the effects of MCC, corresponding to values of $C_{i}$, on the duck curve are presented in Fig 1 . For each of the seasons, several values of $C_{i}$ load curves are presented. For each value of $C_{i}$, the load curve corresponds to the one acquired from the presented model in the following way. Firstly, the minimal value of objective given in Eq. (1) $R$, related to the rampup requirements is calculated by solving the model. In the next step, a schedule is found that maximizes the total provided charge, given in Eq. (2) having the ramp-up requirements equal to the value $R$. This is achieved by adding the following constraint to the model

$$
\sum_{t \in \mathcal{T}_{0}} c_{t}^{2}=R
$$

Since the focus of the first part of the experiments is on the relation between the MCC and the smoothening of the duck curve, the amount of satisfied customer demand is not considered and will be presented in the next subsections. The load curves for different values of the MCC can be seen in Fig. 1. From the load curves in case of the $C_{10}$, it is evident that there is a very high level of flattening the duck curve. In case of low values of MCC $C_{0}$, although there is a notable level of decrease in the steep of the load curve, it is significantly lower than in case of $C_{10}$. The main reason is that a low MCC has a limited impact on "filling" the valley and consequently does not manage to greatly lower the ramp-up requirements.

In case of the selected EV penetration, the MCC needed to reach near maximal levels of flattening the duck curve is significantly lower than the upper bound and is 
nearly three times the lower bound for the MCC. The lowest value of the MCC needed to achieve this is during summer, while it is the highest in winter. It is noteworthy that these results cannot be directly extended to arbitrary regions, since California has mild winters and very hot summers which greatly impact the net demand curve.

\subsection{Satisfaction of requested charges}

In this subsection, we focus on the effect of different levels of satisfied demand on the shape of the duck curve. This is evaluated for different values of the MCC for each season. The upper bound $U_{s}$ for the level of satisfied charge for each MCC is calculated by solving the QP objective given in Eq. (2). Note that, this value is generally lower than the total sum of requested charges for all the EVs. The lower bound $L_{s}$ corresponds to the load curves used in the previous subsection. More precisely, the maximal amount of satisfied requests for the minimal value of the ramp-up related objective given in Eq. (2).

Similar to the previous section, we introduce the following notation for different levels of satisfied charge rate,

$$
S_{i}=L_{s}+\frac{i}{N}\left(U_{s}-L_{s}\right)
$$

In Eq. 13, $N$ is used to indicate the number of different levels of satisfied requests (LSR). The notation $S_{i}$ is used for the $i$ th tested satisfaction level, where $i$ is a value between 0 and $N$. In our tests, $N$ is set to 10 .

A comparison of the effect of different values of the LSR, corresponding to values of $S_{i}$, on the duck curve are presented in Figures 2 and 3 . For each value of $S_{i}$ the load curve corresponds to the charging schedule acquired from the presented model. More precisely, it is the schedule that minimizes the objective, given in Eq. (1) having the total received charge equal to the value $S_{i}$. This is achieved by adding the following constraint to the model

$$
\sum_{t \in \mathcal{T}} l_{t}=S_{i}
$$

The first thing that can be observed from Figures 2 and 3 is that in case of the maximal MCC, there is no significant difference for different values of $S_{i}$. The main reason for this is that there are minuscule benefits to flattening the duck curve by lowering 
the amount of total provided charge. On the other hand, in case of the lower bound for MCC $\left(C_{0}\right)$, the situation is drastically different. In case of $S_{10}$, maximal provided charge, there is a significant increase in the values of the morning and afternoon peak loads. The reason for this is that due to the low level of the MCC, a substantiation amount of requested charge needs to be provided during peak demand periods. This type of behavior is noticeable for all seasons. In case of mid range values of $C_{i}$, similar behavior can be observed but to a lesser extent. Such negative effects can be avoided by dropping a certain amount of provided charge. It is important to note that in cases in which the peak load is increased and the total provided load is decreased, the evening peak demand happens later when compared to the base demand curve. Consequently, this produces a small decrease in ramp-up requirements.

\subsection{Ramp-up and Satisfaction of Charging Requests}

In this subsection, the analysis is focused on the relation between the ramp-up requirements and the amount of satisfied charge requests. This is jointly done for different values of the MCC. In the general case, the best method for evaluating the relation between two objectives is by exploring the Pareto Front.

The presented QP model has proven to well represent the problem of scheduling EV charging for flattening the duck curve. The issue with using this model, for the relation of interest, is that the objective given in Eq (1) is an abstraction that does not directly reflect the ramp-up requirements. When analyzing the need for an increase in electricity production, the most interesting region is the time period 3 hours before the evening peak load. This could relate to an objective of minimizing the difference between total loads at the beginning and ending points of this period. The problem with using such an objective in the presented model is that it generates schedules having some undesirable properties for real world systems (e.g., frequent changes in the sign of the derivative of the load curve). The used quadratic objective given in Eq. (1) avoids this problem.

Due to preceding facts, when exploring the relation between ramping requirements and satisfied charging requests, the following approach is used. Firstly, the Pareto Front is calculated for the two objectives given in Eqs. (1), (2). This has been done, using the 
standard $\epsilon$-constraint method [31]. In the next step, for the solutions corresponding to elements of the Pareto Front the ramp-up requirements, over the relevant time period, have been calculated as the difference between the minimal and maximal total load.

The relevant time period varies between seasons of the the year, due to the length of the day and electrical consumption patterns. Because of this, we choose to use the time periods considered most relevant by California ISO for evaluating ramp-up, for the dates used in generating test instances. It should be noted that these time periods, would vary for an hour between summer and winter.

To jointly analyze the interplay between ramping needs and the customer demand satisfaction for different values of the MCC, the following approach is used in the presentation of results (Figure 4). The ramp-up requirements for each schedule are given relatively to the corresponding value for the base load curve (the net demand without the energy used for EV charging). Consequently, this value ranges from 0 to 1. The value for the satisfaction of requested charges is given relative to the maximal possible one. To be more precise, the maximal amount of satisfied requested charge is the total amount of provided charge (Eq. (2)) for the case where MCC was equal to the upper bound. These results are presented in separate subfigures for each season. Each subfigure contains separate Pareto Fronts for different values of the MCC.

From the presented results, the following conclusions are made. As expected, in case of low values of the MCC, there is a significant decrease in the amount of satisfied requests. Further, for such values of the MCC, there is a need for dropping additional charging requests to achieve a greater decrease in the ramp-up requirements. In case of larger values of the MCC, that are still significantly lower than the upper bound, this becomes less relevant and even if a relatively small amount of charging requests are dropped a notable decrease in ramp-up requirements is achieved. Another interesting property that can be observed for some high values of the MCC in case of summer, is that the ramp-up requirements are even lower than in the case of the upper bound for the MCC. This can be explained with the fact that the time of peak load is shifted for a later time period. This indicates that the objective function given in Eq. (1) could be improved to avoid these types of issues. 


\section{Conclusion}

In this paper, an extensive analysis to investigate the potential contribution of EV demand scheduling to flatten the duck curve has been presented. The scheduling frame-

[1] M. Obi, R. Bass, Trends and challenges of grid-connected photovoltaic systems-a review, Renewable and Sustainable Energy Reviews 58 (2016) 1082-1094.

[2] P. Denholm, M. O'Connell, G. Brinkman, J. Jorgenson, Overgeneration from solar energy in california. a field guide to the duck chart, Tech. rep., National Re-

work has been developed through the use of a QP model. The goal of the analysis was to show the relation between the maximal charging capacity of the facility, level of satisfied requested demands and power grid ramp-up requirements. The investigation has been carried out through a case study based on data of individual charging sessions of EVs from a university campus and real energy consumption data in California. From Firstly, near maximal levels of flattening the duck curve can be achieved by MCC values that are 2 to 3 times higher than the ones corresponding to the minimal one based on the total requested charge. Secondly, although EV charging schedules for lower values of the MCC can decrease the ramp-up requirements, unless some additional charging requests are dropped, there can be a significant increase in peak loads. For higher values of the MCC, a decrease in the satisfaction of requested charges, compared to the maximal possible level, can decrease the ramp-up requirements. This indicates that these types of drops will be necessary in the real world applications of such systems.

The results of the conducted computational experiments provided some insight in designing public charging infrastructures. A natural direction for extending this research is exploring financial aspects of such systems, more precisely the relation infrastructure cost and the effect of operational costs and profits. Another direction would be improving the model by having the maximal charging speed of an EV dependent on the current battery state. newable Energy Lab.(NREL), Golden, CO (United States) (2015). 
[3] M. A. Zehir, A. Batman, M. Bagriyanik, Review and comparison of demand response options for more effective use of renewable energy at consumer level, Renewable and Sustainable Energy Reviews 56 (2016) 631-642.

[4] D. Meyer, J. Wang, Integrating ultra-fast charging stations within the power grids of smart cities: a review, IET Smart Grid 1 (1) (2018) 3-10.

[5] I. Rahman, P. M. Vasant, B. S. M. Singh, M. Abdullah-Al-Wadud, N. Adnan, Review of recent trends in optimization techniques for plug-in hybrid, and electric vehicle charging infrastructures, Renewable and Sustainable Energy Reviews 58 (2016) 1039-1047.

[6] $\mathrm{X}$. Wu, Role of workplace charging opportunities on adoption of plug-in electric vehicles-analysis based on GPS-based longitudinal travel data, Energy Policy 114 (2018) 367-379.

[7] B. Ferguson, V. Nagaraj, E. C. Kara, M. Alizadeh, Optimal planning of workplace electric vehicle charging infrastructure with smart charging opportunities, 355 in: 2018 21st International Conference on Intelligent Transportation Systems (ITSC), IEEE, 2018, pp. 1149-1154.

[8] R. S. Levinson, T. H. West, Impact of convenient away-from-home charging infrastructure, Transportation Research Part D: Transport and Environment 65 (2018) 288-299.

[9] P. J. Tulpule, V. Marano, S. Yurkovich, G. Rizzoni, Economic and environmental impacts of a PV powered workplace parking garage charging station, Applied Energy 108 (2013) 323-332.

[10] Y. Zhang, L. Cai, Dynamic charging scheduling for ev parking lots with photovoltaic power system, IEEE Access 6 (2018) 56995-57005. doi:10.1109/ ACCESS.2018.2873286.

[11] R. Dhawan, S. Prabhakar Karthikeyan, An efficient EV fleet management for charging at workplace using solar energy, in: 2018 National Power Engineering Conference (NPEC), 2018, pp. 1-5. doi : 10.1109/NPEC. 2018.8476746. 
[12] Z. Wei, Y. Li, Y. Zhang, L. Cai, Intelligent parking garage EV charging schedul-

[19] N. Chen, C. W. Tan, T. Q. S. Quek, Electric vehicle charging in smart grid: Optimality and valley-filling algorithms, IEEE Journal of Selected Topics in Signal Processing 8 (6) (2014) 1073-1083. doi:10.1109/JSTSP.2014.2334275

[20] Y. Nakahira, N. Chen, L. Chen, S. H. Low, Smoothed least-laxity-first algorithm 395 ing considering battery charging characteristic, IEEE Transactions on Industrial Electronics 65 (3) (2018) 2806-2816. doi:10.1109/TIE.2017.2740834

[13] K. Jhala, B. Natarajan, A. Pahwa, L. Erickson, Coordinated electric vehicle charging for commercial parking lot with renewable energy sources, Electric Power Components and Systems 45 (3) (2017) 344-353.

[14] R. Jovanovic, I. S. Bayram, Scheduling electric vehicle charging at park-and-ride facilities to flatten duck curves, in: 2019 IEEE Vehicle Power and Propulsion Conference (VPPC), 2019, pp. 1-5.

[15] M. Chertkov, V. Chernyak, Ensemble of thermostatically controlled loads: Statistical physics approach, Scientific reports 7 (1) (2017) 1-9.

[16] H. Hao, B. M. Sanandaji, K. Poolla, T. L. Vincent, Aggregate flexibility of thermostatically controlled loads, IEEE Transactions on Power Systems 30 (1) (2014) 189-198.

[17] F. Sallabi, K. Shuaib, M. Alahmad, Online scheduling scheme for smart electric vehicle charging infrastructure, in: 2017 13th International Wireless Communications and Mobile Computing Conference (IWCMC), 2017, pp. 1297-1302. doi:10.1109/IWCMC.2017.7986472

[18] Junhao Lin, Ka-Cheong Leung, V. O. K. Li, Online scheduling for vehicleto-grid regulation service, in: 2013 IEEE International Conference on Smart

1 Grid Communications (SmartGridComm), 2013, pp. 43-48. doi:10.1109/ SmartGridComm.2013.6687931.

for EV charging, in: Proceedings of the Eighth International Conference on Future Energy Systems, 2017, pp. 242-251. 
[21] Z. J. Lee, T. Li, S. H. Low, ACN-data: Analysis and applications of an open EV charging dataset, in: Proceedings of the Tenth ACM International Conference on Future Energy Systems, 2019, pp. 139-149.

[22] M. K. Gray, W. G. Morsi, Power quality assessment in distribution systems embedded with plug-in hybrid and battery electric vehicles, IEEE Transactions on Power Systems 30 (2) (2014) 663-671.

[23] P. Richardson, D. Flynn, A. Keane, Impact assessment of varying penetrations of electric vehicles on low voltage distribution systems, in: IEEE PES General Meeting, IEEE, 2010, pp. 1-6.

[24] S. H. Baik, Y. G. Jin, Y. T. Yoon, Determining equipment capacity of electric vehicle charging station operator for profit maximization, Energies 11 (9) (2018) 2301.

[25] C. Develder, N. Sadeghianpourhamami, M. Strobbe, N. Refa, Quantifying flexibility in EV charging as DR potential: Analysis of two real-world data sets, in: 2016 IEEE International Conference on Smart Grid Communications (SmartGridComm), IEEE, 2016, pp. 600-605.

[26] M. G. Flammini, G. Prettico, A. Julea, G. Fulli, A. Mazza, G. Chicco, Statistical characterisation of the real transaction data gathered from electric vehicle charging stations, Electric Power Systems Research 166 (2019) 136-150.

[27] Z. Chen, Z. Zhang, J. Zhao, B. Wu, X. Huang, An analysis of the charging characteristics of electric vehicles based on measured data and its application, IEEE Access 6 (2018) 24475-24487.

[28] Y.-W. Chung, B. Khaki, C. Chu, R. Gadh, Electric vehicle user behavior prediction using hybrid kernel density estimator, in: 2018 IEEE International Conference on Probabilistic Methods Applied to Power Systems (PMAPS), IEEE, 2018, pp. 1-6.

[29] Pecan Street Inc, Pecan street dataport (2019). URL https://dataport.cloud 
[30] California ISO, California ISO (2019).

URL http://www.caiso.com

[31] G. Mavrotas, Effective implementation of the $\epsilon$-constraint method in multiobjective mathematical programming problems, Applied Mathematics and Com-

(1) putation 213 (2) (2009) 455 - 465. doi: https://doi.org/10.1016/j . amc. 


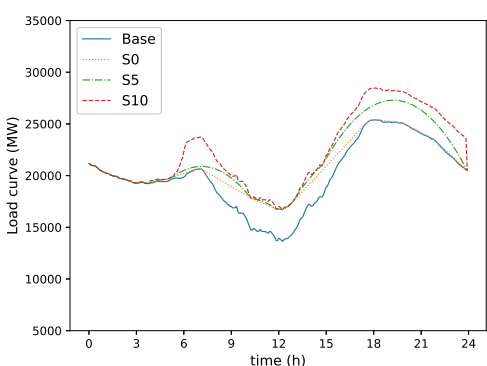

(a) Winter $\mathrm{CO}$

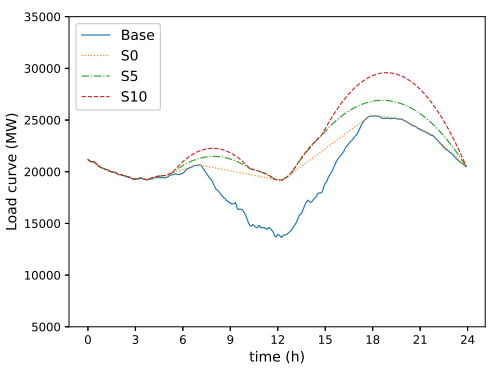

(c) Winter C2

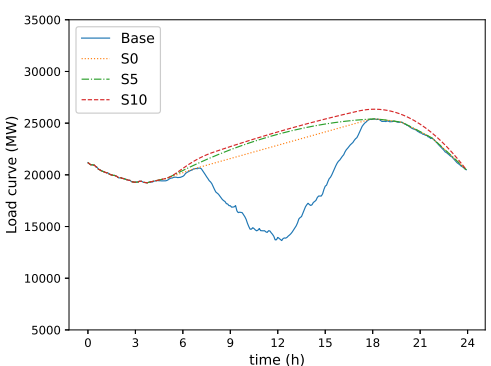

(e) Winter C10



(b) Spring $\mathrm{C} 0$

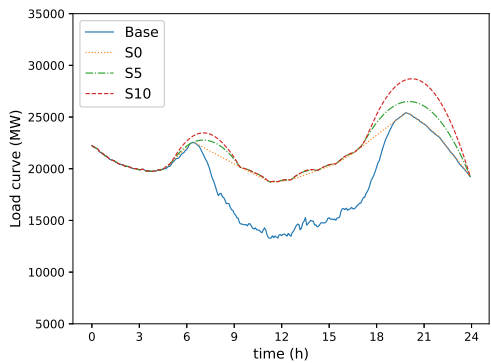

(d) Spring C2

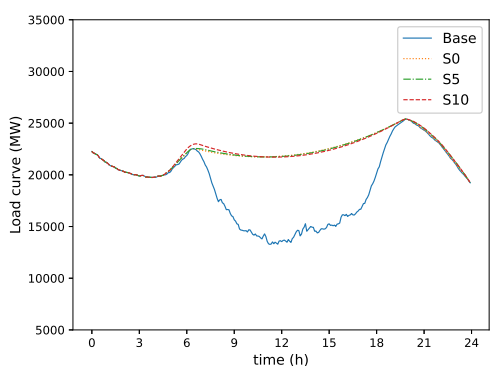

(f) Spring C10

Figure 2: Illustration of the effect of different values of the MCC for different levels of satisfied requested charge by users of the facility. Separate subfigures are used for different values of the MCC in winter and spring. 


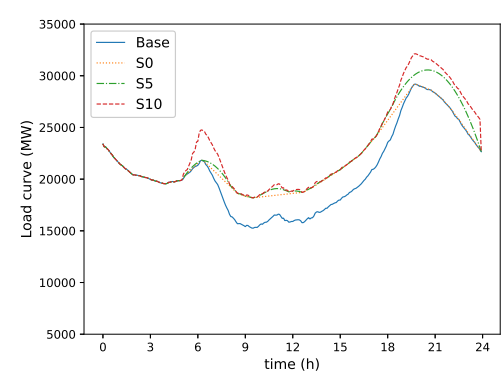

(a) Summer CO



(c) Summer C2

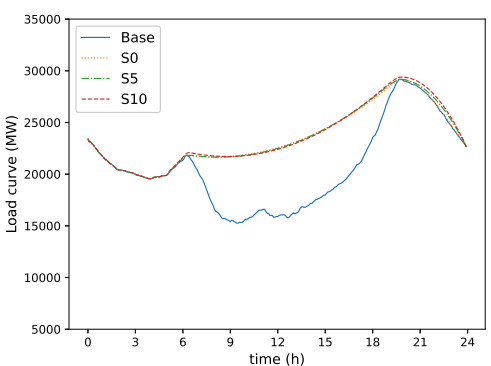

(e) Summer C10

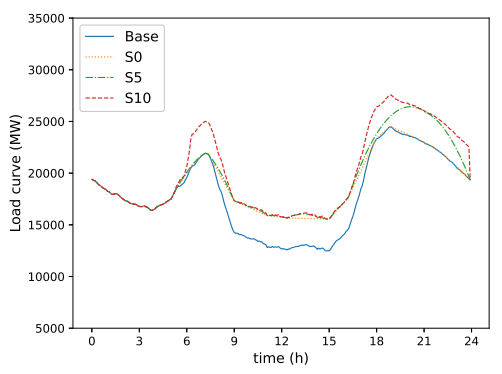

(b) Autumn C0

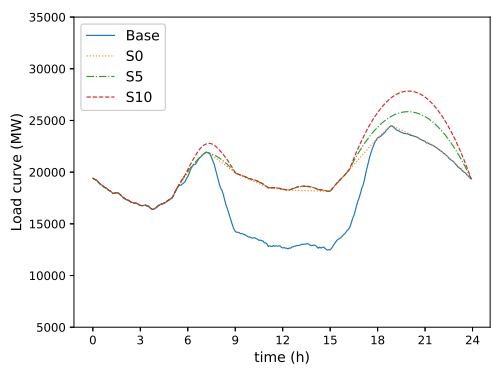

(d) Autumn C2

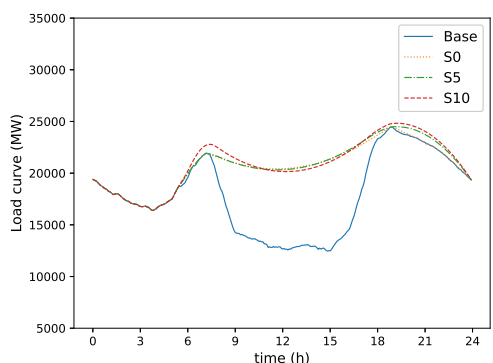

(f) Autumn C10

Figure 3: Illustration of the effect of different values of the MCC for different levels of satisfied requested charge by users of the facility. Separate subfigures are used for different values of the MCC in summer and autumn. 




(a) Winter

(b) Spring

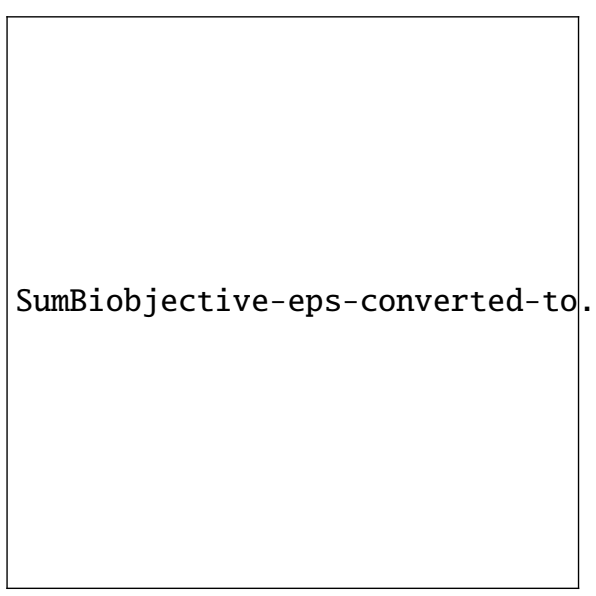

(c) Summer

(d) Autumn

Figure 4: Pareto Fronts for the two objectives: amount of satisfied charge and ramp-up requirements. Separate subfigures are used for different seasons, and in each one of them separate lines represent Pareto Fronts for a specific value of the MCC. The value of the ramp up requirements are given relative to this value for the base load curve. In case of the base load, average ramp up over three hours for winter, spring, summer and autumn, where $11.2 \mathrm{GW}, 8.98 \mathrm{GW}, 9.01 \mathrm{GW}$ and $9.80 \mathrm{GW}$, respectively. 